Diabetologia (1991) 34 [Suppl 1]: S113-S117

0012186X9100126B

\title{
Follow-up study of sensory-motor polyneuropathy in Type 1 (insulin-dependent) diabetic subjects after simultaneous pancreas and kidney transplantation and after graft rejection
}

\author{
W. Müller-Felber ${ }^{1}$, R. Landgraf ${ }^{1}$, St. Wagner ${ }^{1}$, N. Mair $^{1}$, J. Nusser ${ }^{1}$, M. M. C. Landgraf-Leurs ${ }^{1}$, A. Abendroth ${ }^{2}$, \\ W.-D. Illner ${ }^{2}$ and W. Land ${ }^{2}$ \\ ${ }^{1}$ Department of Internal Medicine "Innenstadt", ${ }^{2}$ Transplantation Centre, University of Munich, FRG
}

Summary. The influence of successful simultaneous pancreas and kidney transplantation on peripheral polyneuropathy was investigated in 53 patients for a mean observation period of 40.3 months. Seventeen patients were followed-up for more than 3 years. Symptoms and signs were assessed every 6 months using a standard questionnaire, neurological examination and measurement of sensory and motor nerve conduction velocities. While symptoms of polyneuropathy improved (pain, paraesthesia, cramps, restless-legs) and nerve conduction velocity increased, there was no change of clinical signs (sensation, muscle-force, tendon-reflexes). Following kidney-graft-rejection there was a slight decrease of nerve conduction verlocity during the first year, which was not statistically significant. Following pancreas-graft rejection there was no change of nerve conduction velocity during the first year. Comparing the maximum nerve conduction velocity of the patients with pancreas-graft-rejection to the nerve conduction velocities of these patients at the end of the study, there was a statistically significant decrease of 6.5 $\mathrm{m} / \mathrm{s}$.

In conclusion, we believe that strict normalization of glucose metabolism alters the progressive course of diabetic polyneuropathy. It may be stabilized or partly reversed after successful grafting even in longterm diabetic patients.

Key words: Polyneuropathy - Diabetes mellitus Pancreas transplantation - Kidney transplantation

\section{Introduction}

The main goal of combined pancreas and kidney grafting in patients with long-standing diabetes mellitus is to improve secondary complications. There is some evidence that strict control of hyperglycaemia may favourably influence the course of diabetic complications such as neuropathy, but data remain conflicting (Dyck et al. 1986; Unger 1982). Pancreatic transplantation allows the normalization of glucose metabolism in most patients (Landgraf et al. 1987). Only few data exist, if this procedure, which is only performed at an advanced stage of the disease, is able to reverse diabetic neuropathy (Van der Vliet et al. 1988; Solders et al. 1987; Kennedy et al. 1990).

The purpose of our study was to investigate the influence of successful pancreas and kidney grafting and of graft rejection on diabetic polyneuropathy in Type 1 insulin-dependent diabetic patients.

\section{Subjects and methods}

Subjects. We examined 53 patients ( 25 male, 28 female) with a mean age of 35.1 years (23 - 53). All patients had long standing Type 1 diabetes mellitus (mean duration 20.8 years) and endstage renal disease with haemodialysis or peritoneal dialysis. They had undergone combined pancreas and kidney grafting. Details such as selection criteria, operative and post-operative management and immuno-suppressive protocols have been published previously (Land et al. 1987). The mean observation period following trans-plantation was 40.3 months (13.4 - 97 months). 
Of these patients 9 lost the the function of their kidney-graft alone or before loosing that of the pancreas graft. Graft rejection occurred at a mean interval of 30.4 months after the transplantation ( 1 - 67.6 months). These patients were followedup for a mean period of 13.9 months $(1-23.6)$ after the reoccurrence of uraemia. They were excluded from the study when they also lost the function of their pancreas graft. This occurred after an average of 20 months ( 4 - 33) following the start of dialysis.

Fourteen patients with isolated loss of function of the pancreas graft were investigated for a mean time of 20.8 months ( 4 - 58.4) after graft rejection. Pancreas graft rejection occurred 17.3 months (0.3 - 59.2) after the transplantation. One patient lost the function of his kidney graft 16 months later.

Methods. Most patients were examined every 6 months following transplantation. The first examination after transplantation, which we chose as a baseline, was performed 4.4 months after the transplantation. By using this method we hoped to get results which were not influenced by the release of uraemia but by the normalisation of blood glucose levels.

Symptoms and signs of polyneuropathy were assessed using a standardized questionnaire and a neurological examination protocol at every visit.

Signs of polyneuropathy were defined as mild, if there was only a diminution or loss of tendon reflexes or an impairment of only one sensory modality at the foot. Signs were moderate, if there was an impairment of more than one sensory modality at the foot or more proximal sensory disturbances. Ataxia or impairment of more than one sensory modality in the proximal segments of the limb was defined as severe polyneuropathy.

Using surface stimulation and registration electrodes motor nerve conduction velocities of the right median and peroneal nerve and sensory nerve conduction velocities of the distal right median and of the distal sural nerve (ankle to foot) were measured. If necessary an averager was used to obtain a sensory action potential.

The parameters used were nerve conduction velocities (NCV), distal latencies (DL) and amplitudes of sensory nerve action potentials (SNAP).

An electromyographic examination was only done at the entry to the study but not during the follow-up period.

Statistical analysis. The statistical analysis of clinical and neurophysiological data was done using the paired t- test. The level of significance was set at $p<0.05$. We compared the intraindividual variation between the first examination and the end of years 1, 2 and, if the grafts survived, year 3. In the patients with isolated loss of function of either the pancreas- or kidneygraft, we compared the data of the last examination before to those $1 / 2$ and 1 year after graft rejection.

\section{Results}

\section{Symptoms and signs of neuropathy}

Before transplantation $61 \%$ of the patients had at least one symptom of neuropathy (Table 1). Symptoms were mild in $62 \%$, moderate to severe in $38 \%$.

Following transplantation symptoms of $8 \%$ of the symptomatic patients remained unchanged, $50 \%$ improved and $42 \%$ became asymptomatic.

Symptoms reoccurred in $22 \%$ of these patients at an interval between 2 and 4 years despite normal graft function.

Table 1: Symptoms of polyneuropathy

\begin{tabular}{lll}
\hline & $\begin{array}{l}\text { Before } \\
\text { transplantation }\end{array}$ & $\begin{array}{l}\text { 2 years after } \\
\text { transplantation }\end{array}$ \\
\hline Pain & $18 \%$ & $12 \%$ \\
Cramps & $26 \%$ & $13 \%$ \\
Paraesthesia & $48 \%$ & $8 \%$ \\
Restless legs & $5 \%$ & $5 \%$ \\
\hline
\end{tabular}

Neurological examination at the beginning of the study was normal in $9 \%$ of the patients. Minimal signs of polyneuropathy were found in $39 \%$, they were moderate in $39 \%$ and severe in $13 \%$. The most common clinical signs were an absent or diminished achilles tendon reflex in $92 \%$ and pallhypaesthesia in $62 \%$. Gait ataxia was seen in 15 $\%$.

There was no significant change of muscle force, sensory deficits or tendon reflexes.

During the follow-up period $26 \%$ of the patients developed symptomatic carpal tunnel syndrome (CTS). CTS was unilateral in $9 \%$, bilateral in $17 \%$. Surgical decompression of the ligament carpi transversum was only necessary in one patient.

\section{Electrophysiological studies}

At the beginning of the study the extensor digitorum brevis muscle (edb) was denervated in $20 \%$ of the patients. No sensory compound action potential of the distal sural nerve could be measured in $69 \%$.

During the follow-up period in one patient reinnervation of the edb was observed, in one patient denervation occurred. There was no reinnervation of the sural nerve. 
During the follow-up period motor peroneal nerve conduction velocities of those patients, whose edb muscle was not denervated, increased by an average of $6.6 \mathrm{~m} / \mathrm{s}(\mathrm{p}<0.002)$ (Fig. 1). The increase was significant after the second year. There was a significant difference of median motor NCVs between the beginning of the study and after 1 year $(4.5 \mathrm{~m} / \mathrm{s})(\mathrm{p}<0.008)$ and after 3 years $(6.8 \mathrm{~m} / \mathrm{s})(\mathrm{p}<$ $0.0002)$ (Fig. 1). The mean sural nerve conduction velocities increased by $6.6 \mathrm{~m} / \mathrm{s}$ between the first examination and three years after the transplantation (Fig. 1). Due to the small number of patients without denervation of this nerve, the results did not reach the level of significance.

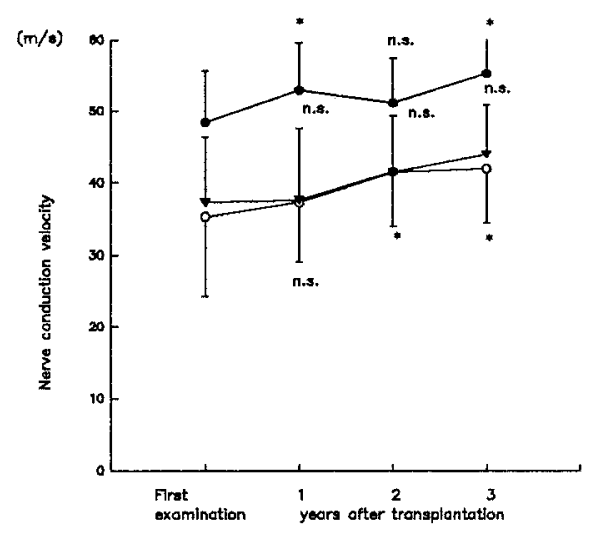

Fig. 1: Nerve conduction velocities in patients with functioning pancreas and kidney grafts $(*=p<0.05$, n.s. $=p>0.05)(\bullet=$ median motor nerve, $o=$ peroneal motor nerve, $v=$ sural nerve)

NCVs of the sensory median nerve did not increase significantly. The patients with symptoms of a carpal tunnel syndrome showed a decrease of the sensory NCVs and an increase of the distal latencies.

The amplitudes of the sensory nerve action potential did not increase significantly.

\section{Graft rejection}

After kidney graft rejection symptoms reoccurred in $30 \%$ of the patients. The mean decrease of peroneal nerve conduction velocity after 1 year was $10.2 \mathrm{~m} / \mathrm{s}$ and of the motor median nerve conduction velocity $9.2 \mathrm{~m} / \mathrm{s} \mathrm{(Fig.} \mathrm{2).} \mathrm{Due} \mathrm{to} \mathrm{the} \mathrm{small} \mathrm{number} \mathrm{of} \mathrm{patients}$ this was not statistically significant.

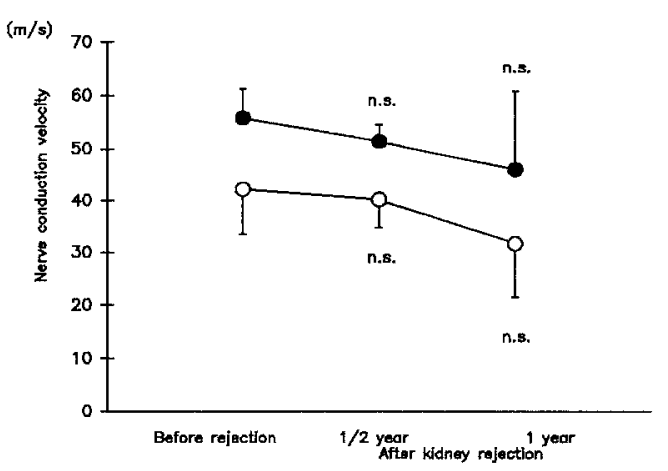

Fig. 2: Peroneal and median NCVs following kindney graft rejection $\left({ }^{*}=\mathrm{p}<0.05\right.$, n.s. $\left.=\mathrm{p}>0.05\right)(\bullet=$ median motor nerve, $o=$ peroneal motor nerve)

Following pancreas graft rejection in $31 \%$ of the patients symptoms reoccurred or worsened. Despite rejection of the pancreas graft there was a mean increase of peroneal nerve conduction velocities of $4.9 \mathrm{~m} / \mathrm{s}$, which was not statistically significant. Median nerve conduction velocities decreased at an average of $1.5 \mathrm{~m} / \mathrm{s}$. (Fig. 3).

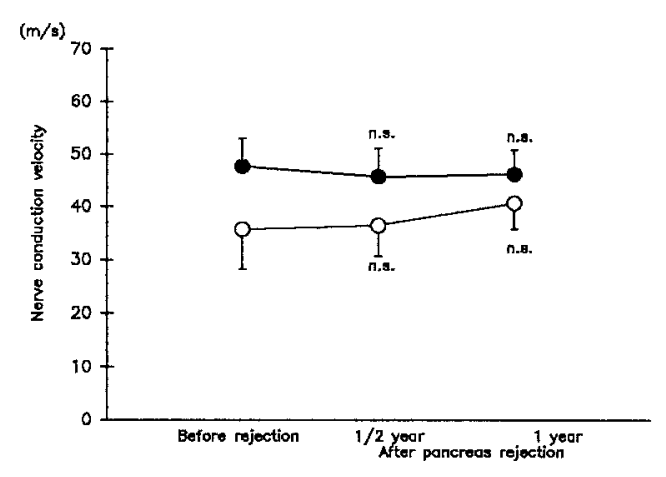

Fig. 3: Peroneal and median NCVs following pancreas graft rejection $\left({ }^{*}=\mathrm{p}<0.05\right.$, n.s. $\left.=\mathrm{p}>0.05\right)(\bullet=$ median motor nerve, $o=$ peroneal motor nerve)

Despite the fact, that in some patients nerve conduction velocities improved during the first year following pancreas graft rejection, in all but one patient a decrease of NCVs occurred at variable intervals after the loss of graft function. Comparing the peroneal NCV before pancreas graft rejection or the maximum NCV following graft rejection in those cases who continued to improve despite loss of pancreas function, to the NCV at the end of the study, there was a statistically significant decrease of 
$6.5 \mathrm{~m} / \mathrm{s}(\mathrm{p}<0.002)$. The worsening of the NCVs began at very different intervals from the time of rejection (0 to 51 months).

\section{Discussion}

Following simultaneous pancreas and kidney graft transplantation symptoms of polyneuropathy improved in our group of patients whereas neurological signs remained unchanged. In contrast to the natural course of diabetic peripheral neuropathy (Boulton et al. 1983) subjective claims of our patients, even those existing before the manifestation of uraemia, improved markedly. It is undecided, whether this is only a psychological effect or really a change of nerve function. Concerning the neurological status our data confirm the results of a previous study examining the effect of isolated pancreas transplantation in nonuraemic diabetic patients (Van der Vliet et al. 1988).

The fact that we did not observe any change of neurological signs may be due to two different reasons:

1) it may indicate, that the structural lesion in diabetes is irreversible.

2) changes may be too small to be seen within the follow-up period. Unfortunately there is a methodological problem to be overcome: using sophisticated methods to assess sensory and motor deficits such as psychophysic testing of thermal or pain thresholds there is a great intraindividual variation due to factors such as alertness and cooperation. The more precise a clinical examination protocol is, the less reliable it is (American Diabetes Association 1988). Using a simple grading of neuropathic signs small changes cannot be seen.

Nerve conduction velocities of the upper and lower extremities increased significantly in our study. The same results have been obtained in three other studies (Van der Vliet et al. 1988; Solders et al. 1987; Kennedy et al. 1990). In both studies the authors compared the NCVs before transplantation to that 2 years after transplantation.

In contrast to these studies we took the status after the transplantation as a baseline for the following reasons:

1) changes between the status before and after transplantation may be only, or to a large extent, the result of resolving uraemia. Because the type and severity of polyneuropathy differ between diabetic and non-diabetic patients (Solders et al. 1987), using non-diabetic kidney transplanted patients as a control cannot solve this problem.

2) The time interval between pre-transplant examination and transplantation varies widely since patients were examined neurologically some weeks or 1 to 2 years prior to transplantation. The severity of polyneuropathy changes unpredictably during this time.

The fact that nerve conduction velocities improved over a period of three years, as we have shown in our study, indicates that there may be a structural change of the nerve. On the other hand the amplitudes of sensory nerve action potentials, which correlate to the number of axons of the nerve, remained unchanged. Reinnervation of denervated nerves was not observed except in one case. These results confirm the data of van der Vliet et al (1988) and this may indicate that the axonal dammage is irreversible while there is some change of the myelin sheath.

The effect of graft rejection has not been examined as yet by other groups. As far as our data show, rejection of the kidney leads to a rather immediate decrease of nerve conduction velocities. The fact that the maximal change is seen within a few months may indicate that this is caused by functional rather than by morphological changes.

The opposite was seen in patients with loss of function of the pancreas graft. There was no shortterm effect but a decrease of nerve conduction velocity at variable intervals after rejection.

Thus, our data as well as the data mentioned above suggest that strict normalization of glucose metabolism alters the progressive course of diabetic polyneuropathy.

In conclusion, we believe that polyneuropathy may be stabilized or partly reversed after successful grafting even in long-term-diabetic patients.

\section{References}

American Diabetes Association (1988): Report and recommendations of the San Antonio conference on diabetic neuropathy. Muscle and Nerve 11: $661-667$

Boulton A.J.M., Scarpello J.H.B., Armstrong W. D., Ward J.D. (1983): The natural history of painful diabetic neuropathy- a 4 year study. Postgraduate Medical J. 59: 556 - 559

Dyck P.J., Brown M., Greene D. (1986): Does improved control of glycaemia prevent or ameliorate diabetic polyneuropathy ? Ann. Neurol. 19: $288-293$

Kennedy R.W., Navarro X., Goetz F.C., Sutherland D.E., Najarian S. (1990): Effects of pancreatic transplantation on diabetic neuropathy. N Engl J of Med 322: 1031 - 1037 
W. Müller-Felber et al.: Polyneuropathy after pancreas grafting and after graft rejection

Land W., Landgraf R., Illner W, Abendroth D., Kampik A., Jensen U., Lenhart F.P., Burg D., Hillebrand, G., Castro LA, Landgraf-Leurs MMC, Frey L., Gokel M., Schleibner H.,

Nusser J., Ulbig M. (1987): Clinical pancreatic transplantation using the prolamine duct occlusion technique- The Munich Experience. Transpl. Proc. 19 (Suppl. 4): 75 - 83

Landgraf R., Landgraf-Leurs MMC, Kampik A., Burg D., Castro LA., Abendroth D., Illner WD., Land W. (1987): Pancreas transplantation as a mean for insulin replacement: An outlook. In: P. Brunetti, W.K. Waldhäusl (eds) Advanced Models for the Therapy of Insulin-Dependent Diabetes. Raven Press Vol. 37:395 - 400

Solders G., Gunnarsson R., Persson A., Wilczek H., Tyden G., Groth C.G (1987): Effects of combined pancreatic and renal transplantation on diabetic neuropathy: a two-year follow-up study. The Lancet 333: 1232 - 1235

Unger R.H. (1982): Meticulous control of diabetes: benefits, risks and precautions. Diabetes 31: $479-483$

Van der Vliet J.A., Navarro X., Kennedy W.R, Goetz F.C, Najarian J.S., Sutherland D.E. (1988): The effect of pancreas transplantation on diabetic polyneuropathy. Transplantation 45: 368 - 370

Dr. W. Müller-Felber

Friedrich-Baur-Institut

Ziemssenstr. 1 a

8000 München 2

FRG 